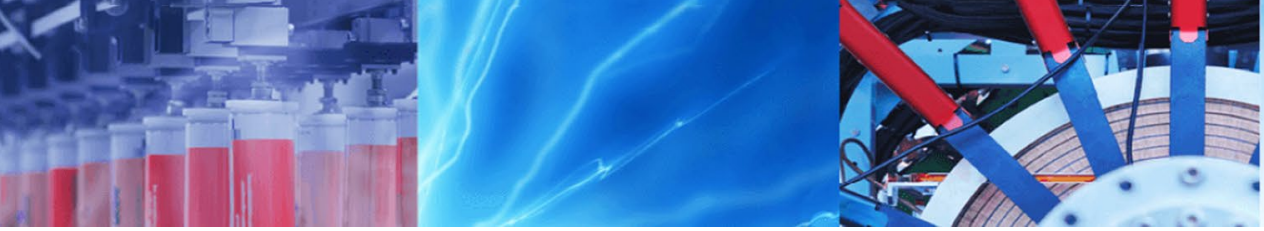

Research Article

\title{
Multi-view representation learning for segmentation of abnormal tissues in medical images applied to multiple sclerosis lesion delineation
}

\author{
Hassan Khastavaneh $^{1}$ (1) $\cdot$ Hossein Ebrahimpour-Komleh $^{1}$ (1)
}

C Springer Nature Switzerland AG 2019

\begin{abstract}
Automated segmentation of abnormal tissues in medical images assists both physicians and medical researchers in the process of diseases diagnostic and research activities respectively. Intelligent techniques of automated segmentation are gaining more popularity in contrast to non-intelligent ones. In these techniques, quality representation of pixel/voxels by considering multiple natural and artificial views which exist in medical images increases segmentation accuracy. The proposed method for segmentation of abnormal tissues in medical images is based on multi-view representation with six phases of pre-processing, view generation, representation generation, classification, post-processing, and evaluation. In the representation phase, raw data of medical images are represented based on the modes of variation or clusters exist in the original multi-view feature space. Quantitative results of the experiment demonstrate representations generated via the proposed method are effective especially when the Random Forest classifier is employed. DSC of 0.72 for a subject shows that the results are promising. This study shows cluster based representation of raw pixel/voxels of multiple views are effective in supervised segmentation of abnormal tissues.
\end{abstract}

Keywords Medical imaging · Abnormal tissue segmentation · Multiple sclerosis lesions · Multi-view representation learning

\section{Introduction}

Image segmentation refers to the partitioning of digital images into distinct regions in a way that each region has its own visual characteristics or computed properties. Medical images as a broad category of digital images utilize automated segmentation techniques for separating anatomical structures or delineating abnormalities appeared in the images of different organs of human body. Automatic delineation assists both physicians and medical researchers while diagnosing diseases, studying anatomical structures, and developing new drugs.

Multiple sclerosis (MS) is considered as a demyelinating diseases of central nervous system in which the myelin shields of nerve cells are damaged. MS lesions appear as hyper-intense and hypo-intense signals in different modalities of magnetic resonance images. Volume and count of these lesions are important biomarkers for diagnosis and control of MS. Since manual segmentation of the lesions by trained radiologist is time consuming, cumbersome, error-prone and subjective; automatic segmentation is preferred. The most accurate automatic segmentation methods attempt to utilize machine learning techniques for modeling normal appearing brain tissues and lesion areas in either supervised or unsupervised fashion. Both supervised and unsupervised methods use variety of features including, intensity, spatial location, shape, size, texture [1], and atlas features. Recently, deep neural networks have been applied on the task of MS lesion segmentation. Location sensitive deep neural networks consider

Hassan Khastavaneh, khastavaneh@hotmail.com | 'Department of Computer Engineering, University of Kashan, Kashan, Esfahān, Iran.

SN Applied Sciences (2019) 1:1084 | https://doi.org/10.1007/s42452-019-1151-7

Received: 21 May 2019 / Accepted: 22 August 2019 / Published online: 24 August 2019 
the location of lesions on the decision making process [2]; other deep networks utilize shortcut connections for better performance [3].

Intelligent methods of abnormal tissue segmentation, regardless of being supervised or unsupervised, have two important phases of feature extraction and feature selection prior to the modeling phase. As the quality of generated features is very important for consequent modeling, both feature extraction and feature selection phases are very important in the pipeline of intelligent abnormal tissue segmentation.

Representation learning techniques are approximately a new paradigm in feature generation, attempts to generate features by learning representations directly from the data [4]. Representation learning techniques are able to extract and abstract key information from medical images.

In this study, a supervise pixel/voxel-based segmentation method with the aid of multi-view representation learning paradigm is proposed. As medical images can be acquired using different modalities with different parameters, multiple images of an organ under analysis are potentially available. Utilizing all of these images in the process of abnormal tissue modeling potentially leads to better performance on the segmentation results. Extracting information from multiple image modalities is referred as multi-view representation learning which has strong relation to the topic of multi-view learning [5]. Multi-view representation learning is also effective in cases that the views are artificially generated and derived from real views which themselves are the result of measurements performed by sensors. The proposed method abstract information exist in multiple views via an unsupervised representation learning approach. Extracted information are used to classify individual pixel/voxels as belonging to either normal or abnormal tissues.

Rest of this paper is organized as follows: the proposed method is explained in Sect. 2. Section 3 explains experimental results. And finally, the paper is concluded in Sect. 4.

\section{Materials and methods}

\subsection{Dataset}

In order to evaluate performance of the proposed segmentation method, a benchmark and publicly available dataset related to the segmentation of MS lesions is considered. As newest datasets address new difficulties, challenges, and evaluation protocols; in this study, one of the recently published dataset is utilized. For each subject in the dataset, T1w, contrast-enhanced T1w, T2w, and FLAIR modalities are available [6].

SN Applied Sciences

\subsection{Method}

In this section, the proposed method for segmentation of abnormal tissues in medical images is explained. The proposed method attempts to consider information obtained from multiple real or artificial views of medical images. The values of a typical pixel/voxel in different views are abstracted by utilizing modes of variations in the data. After exploring modes of variation or clusters in the data, each pixel/voxel is represented based on the identified modes.

The proposed method has six phases of pre-processing, view generation, representation generation, classification, post-processing, and evaluation. In the following sections, the goal and functionality of each phase is explained.

\subsubsection{Pre-processing}

In the pre-processing phase, some processes on the raw images are applied to prepare them for better performance on the main task of segmentation. Common preprocessing tasks of MR images for segmentation of MS lesions are brain extraction [7], bias field correction [8], registration [9], and intensity normalization. Intensity of voxels inside the brain mask for all modalities are re-scaled into the range $[0,255]$ using min-max normalization as described by Eq. 1 .

Intensity $_{\text {new }}=\frac{\text { Intensity }_{\text {old }}-\text { Intensity }_{\min }}{\text { Intensity }_{\max }-\text { Intensity }_{\min }} .255$

\subsubsection{View generation}

View generation phase prepare and generate multiple views from the data. These views are categorized into two types of natural and artificial. Natural views are those which are measured directly via sensors; artificial views are those which are computed from natural views to give more insights about the data. With these explanations, the dataset used in this study has two natural views corresponding to T2-W and FLAIR MR modalities; in order to enrich views, 7 artificial views are generated. Both artificial and natural views are summarized in Table 1.

\subsubsection{Representation generation}

In the representation generation phase, multiple views which indeed explain a voxel inside the brain volume with different perspectives are abstracted into more representative and informative set of features. These features are later used for classifying individual pixel/ voxels as belonging to either normal or abnormal 
Table 1 Summary of different natural and artificial views which describe each voxel

\begin{tabular}{|c|c|}
\hline $\begin{array}{l}\text { View } \\
\text { number }\end{array}$ & Description \\
\hline 1 & T2-w modality \\
\hline 2 & FLAIR modality \\
\hline 3 & $\begin{array}{l}\text { Average intensity in } 3 \times 3 \times 3 \text { neighborhood of T2-w } \\
\text { modality }\end{array}$ \\
\hline 4 & $\begin{array}{l}\text { Average intensity in } 3 \times 3 \times 3 \text { neighborhood of FLAIR } \\
\text { modality }\end{array}$ \\
\hline 5 & $\begin{array}{l}\text { Average intensity in } 5 \times 5 \times 5 \text { neighborhood of T2-w } \\
\text { modality }\end{array}$ \\
\hline 6 & $\begin{array}{l}\text { Average intensity in } 5 \times 5 \times 5 \text { neighborhood of FLAIR } \\
\text { modality }\end{array}$ \\
\hline 7 & Average intensity of whole brain tissue in T2-w modality \\
\hline 8 & Average intensity of whole brain tissue in FLAIR modality \\
\hline 9 & Distance from mid-sagittal plane \\
\hline
\end{tabular}

tissues. Representation generation phase attempts to find modes or clusters hidden in the data and represent each pixel/voxel according to the founded modes by incorporating multiple views.

In order to discover modes of variation, k-means clustering algorithm is employed [10]. This algorithm finds a fixed number of clusters in the data by minimizing cost function described by Eq. 2 . In this equation, $N, m, U, x_{i}$ and $\theta$ are total number of pixel/voxels, total number of clusters, assignment matrix, ith pixel/voxel, and vector of cluster means respectively. An important parameter for k-means clustering algorithm is the number of clusters which depends on the task and the data. In case of MS lesion segmentation, it is set to 8 . The reason for choosing 8 as the number of clusters is that in MS lesion segmentation task, the pixel/voxels inside the brain mask belong to eight compartments of gray matter (GM), white matter (WM), cerebrospinal fluid (CSF), partial volume of GM-CSF, partial volume of WM-GM, lesions, background, and artifacts.

$J(\theta, U)=\sum_{i=1}^{N} \sum_{j=1}^{m} u_{i j}\left\|x_{i}-\theta_{j}\right\|^{2}$

After finding modes of variation hidden in the data, it is time to generate a new representation for each individual pixel/voxel according to the founded cluster centers. In fact, new representation for each pixel/voxel is an 8-dimensional vector whose entries hold distance of vector describing that pixel/voxel to the cluster centers as described by Eq. 3 . In this equation, for each dimension $j$ of $y$ as new representation, Euclidean distance of the ith data point to the $C_{j}$ as jth cluster center is measured; here, $d$ is the dimension of original data. In fact, representation generation phase describes each individual pixel/voxel by 8 cluster center. These distances measure how much a typical pixel/voxel belongs to the different regions of the feature space.

$y_{i}^{j}=\sqrt{\sum_{p=1}^{d}\left(x_{i}^{p}-c_{j}^{p}\right)^{2}} ;$ for $j=1 \ldots m$

\subsubsection{Classification}

Classification phase of the proposed method attempts to build some model for deciding whether a pixel/voxel belongs to either a normal or abnormal tissue. Four classification techniques namely, multi-layer perceptron (MLP), k-nearest-neighbor (KNN), random forest (RF), and logistic regression (LR) are employed to perform classification task.

\subsubsection{Post-processing}

As a result of attempting to keep both sensitivity and specificity of automated segmentation methods in an acceptable level, false positives are inevitable. In order to get-rid-of these false positives, the lesions with the volume less than 25 voxels are removed. This threshold is founded experimentally.

\subsubsection{Evaluation}

In order to quantitatively evaluate performance of the proposed segmentation method, accuracy (ACC), sensitivity (SEN), specificity (SPE), dice similarity coefficient (DSC), area under the curve (AUC), and volume similarity (VS) as standard metrics are utilized. Among these metrics, DSC is commonly used for evaluation of MS lesion segmentation methods; this metric actually measures similarity of two binary set; one for ground truth and the other one for automated segmentation results.

\section{Experimental results}

After successful implementation of the proposed abnormal tissue segmentation method, its performance is measured quantitatively on 10 randomly selected subjects of the dataset. In order to quantitatively measure performance of the proposed method, all of the previously mentioned metrics are calculated for each subject individually. The results for each classifier are averaged over all the subjects and summarized in Table 2. As this table shows, the best classifier is RF.

Although the best result belongs to the RF classifier, all of the other classifiers demonstrate almost close 
Table 2 Average performance of the proposed method for each classifier with various metrics

\begin{tabular}{lllllll}
\hline Classifier & ACC & SEN & SPE & DSC & AUC & VS \\
\hline MLP & 0.88 & 0.93 & 0.88 & 0.30 & 0.91 & 0.33 \\
KNN & 0.91 & 0.97 & 0.91 & 0.37 & 0.94 & 0.39 \\
RF & 0.94 & 0.99 & 0.94 & 0.46 & 0.96 & 0.47 \\
LR & 0.88 & 0.86 & 0.88 & 0.29 & 0.88 & 0.33 \\
\hline
\end{tabular}

performance to it; this closeness revealed the fact that representations generated via the proposed method are stable and consistent. The results also show that the worst classifier is LR because of its linear nature.

Sensitivity of 0.99 for the best-performed classifier shows that majority of abnormal voxels are correctly identified. Although specificity of best-performed classifier is 0.94, but the AUC of 0.96 demonstrate acceptable tradeoff between sensitivity and specificity.

Performance of the proposed method with random forest as the best-performed classifier for each subject in terms of all metrics is calculated; the DSC for best subject is 0.72 which is very promising.

\section{Conclusion}

Representation based on the clustering is a kind of distributed representation. Distributed representation as a requirement for real-world intelligence is the capability which leads each cluster concerns one specific aspect of the data which is presented based on that cluster. Different clustering method and distance measures may improve the results which will be considered in the future works.

\section{Compliance with ethical standards}

Conflict of interest On behalf of all authors, the corresponding author states that there is no conflict of interest.

\section{References}

1. Abdullah B (2011) Textural based SVM for MS lesion segmentation in FLAIR MRIs. Open J Med Imaging 1:26-42
2. Ghafoorian $M$, Karssemeijer N, Heskes $T$, van Uden IWM, Sanchez $\mathrm{Cl}$, Litjens G, de Leeuw F-E, van Ginneken B, Marchiori E, Platel B (2017) Location sensitive deep convolutional neural networks for segmentation of white matter hyperintensities. Sci Rep 7(1):5110

3. Brosch T, Tang LYW, Yoo Y, Li DKB, Traboulsee A, Tam R (2016) Deep 3D convolutional encoder networks with shortcuts for multiscale feature integration applied to multiple sclerosis lesion segmentation. IEEE Trans Med Imaging 35:1229-1239

4. Bengio Y, Courville A, Vincent P (2012) Representation learning: a review and new perspectives. IEEE Trans Pattern Anal Mach Intell 35:1798-1828

5. LiY, Yang M, Zhang Z (2016) Multi-view representation learning: a survey from shallow methods to deep methods. IEEE Trans Knowl Eng 14:1-20

6. Lesjak Ž, Galimzianova A, Koren A, Lukin M, Pernuš F, Likar B, Špiclin Ž (2018) A novel public MR image dataset of multiple sclerosis patients with lesion segmentations based on multirater consensus. Neuroinformatics 16:51-63

7. Iglesias JE, Liu CY, Thompson PM, Tu Z (2011) Robust brain extraction across datasets and comparison with publicly available methods. IEEE Trans Med Imaging 30:1617-1634

8. Tustison NJ, Avants BB, Cook PA, Zheng Y, Egan A, Yushkevich PA, Gee JC (2010) N4ITK: improved N3 bias correction. IEEE Trans Med Imaging 29:1310-1320

9. Klein S, Staring M, Murphy K, Viergever MA, Pluim JPW (2010) Elastix: a toolbox for intensity-based medical image registration. IEEE Trans Med Imaging 29:196-205

10. Theodoridis S, Koutroumbas K (2009) Pattern recognition. Elsevier, Amsterdam

Publisher's Note Springer Nature remains neutral with regard to jurisdictional claims in published maps and institutional affiliations. 